\title{
Pathophysiological mechanisms of alcoholic myopathy - Lessons from rodent models
}

\author{
(iD \\ Danielle E. Levitt' ${ }^{1}$, Patricia E. Molina ${ }^{2}$ and Liz Simon ${ }^{3}$ \\ Department of Physiology, Louisiana State University Health Sciences Center, 1901 Perdido
} Street, New Orleans, Louisiana, USA 70112

Citation: Levitt, D. E., Molina, P. E. and Simon, L. 2021. Pathophysiological mechanisms of alcoholic myopathy: Lessons from rodent models. J. Vet. Ani. Sci. 52 (2): 107-116.

DOI: https://doi.org/10.51966/jvas.2021.52.2.107-116

Received: 07.12.2020

Accepted: 01.01.2021

Published: 01.06.2021

\begin{abstract}
Skeletal muscle dysfunction is highly prevalent and is one of the earliest pathological tissue changes among people with at-risk alcohol use. Clinical studies to elucidate pathophysiological mechanisms of alcohol-mediated muscle disease are hampered due to ethical considerations, and confounded by nutritional, lifestyle, and comorbid conditions. Rodent models have been developed to study the impact of at-risk alcohol consumption and alcohol-mediated end organ injury, including skeletal muscle dysfunction. This review discusses results from well-established rodent models of alcohol administration and highlights key pathophysiological mechanisms underlying alcoholic myopathy identified in rodent models. Salient pathways include impaired regenerative capacity, altered anabolic/catabolic balance, impaired mitochondrial bioenergetic function, and skeletal muscle morphological and contractile changes.
\end{abstract}

Key words: Alcohol, Muscle, Protein synthesis, Stem cell regeneration, Mitochondria

Alcoholic myopathy, or decreased skeletal muscle mass or function, occurs in 40 to 60 percent of people with at-risk alcohol use (Fernandez-Sola et al., 2007; Preedy et al., 2003) and is one of the earliest pathological tissue changes seen with alcohol use (Lang et al., 2005; Martin et al., 1985). Although alcohol-related muscle disease is nearly five times more common than liver cirrhosis (Estruch et al., 1993), mechanistic data are lacking on its contribution to long-term health, aging, as well as its association with injury, metabolic dysregulation, or disuse atrophy. Ethical issues, complexity of alcohol use patterns, and confounding comorbidities make clinical studies difficult and have led to the development of preclinical models, especially rodents, to understand alcohol-mediated pathophysiological mechanisms underlying human disease. There are always concerns and questions regarding the use of animals in biomedical research and especially in substance use research. However, we obtain reliable data, there is great degree of experimental control and replicability, allowing for mechanistic investigation of human drug use and abuse.

1. Postdoctoral fellow

2. Professor and Chair

3. Associate Professor and corresponding author Email: Isimo2@Isuhsc.edu

Copyright: (C) 2021 Danielle E. Levitt et al. This is an open access article distributed under the terms of the Creative Commons Attribution 4.0 International License (http://creativecommons.org/licenses/by/4.0/), which permits unrestricted use, distribution, and reproduction in any medium, provided the original author and source are credited. 


\section{Why study alcohol and end organ injury?}

In the 1930s, E. M. Jellinek, a physiologist, described alcoholism (now referred to as alcohol use disorder; AUD) as a disease. His seminal paper provided clarity and removed stigma associated with AUD, and underscored that it was not due to weakness of will or temperament, moral failing, symptom of mental illness, but that it is a physical illness due to a pharmacologically addictive substance that can affect people from all sections of society (Jellinek and McFarland, 1940). However, at-risk alcohol use has adverse effects on many organ systems, including the liver, lungs, central and peripheral nervous system, immune system, and skeletal muscle. With such wide-ranging effects, it is critical to identify mechanisms leading to alcohol-mediated pathology to better manage and treat alcohol-related disease.

\section{Alcohol administration in rodents}

Several reliable models of alcohol administration in rodents have been developed and there are benefits and drawbacks of each. Here, we briefly describe some of the most common models. Some of the models developed allow for excessive drinking and alcohol-seeking behavior demonstrating the rewarding effects of alcohol and their contribution to escalating alcohol use; others are more commonly used to study end-organ effects of alcohol.

1) Two-bottle choice method: Animals are allowed an unrestricted choice between alcohol and water for a predetermined amount of time up to 24 hours per day. Animals consume alcohol voluntarily. Work with these models show heterogeneous populations of animals, like humans, some of which consume larger volumes of alcohol than others, and can help elucidate how certain factors, such as previous patterns of alcohol administration (Younis et al., 2019; Nentwig et al., 2019), influence alcohol preference. These studies have also shown that genetic manipulation by inbreeding or selective breeding can produce animals displaying different alcohol preferences (Grahame et al., 1999).
2) Operant models: Animals must perform a certain task for alcohol intake. For example, animals are trained to press a lever for alcohol to be delivered through the oral route, or in some cases directly into the brain. These models allow for the assessment of motivation to consume alcohol, such as after traumatic brain injury (Stielper et al., 2019) and to elucidate possible factors underlying motivation for alcohol, such as altered glucocorticoid receptor signaling (Pahng et al, 2019).

3) Intragastric administration: Animals are administered alcohol via infusion directly into the stomach through surgically implanted intragastric tubes. This method is used to avoid the influence of taste and has been used to study alcoholic liver disease; e.g., Tsukamoto-French model (Tsukamoto et al., 1990).

4) Alcohol-containing liquid diet: After transition from solid to liquid diet, alcohol containing liquid diet is the sole source of nutrition in this model. The alcohol content of the liquid diet is increased over the course of several days at the beginning of the experimental period and subsequently maintained at the target level. The most common diet used is the LieberDeCarli diet (Lieber and DeCarli,1989) and the control diet is isocalorically matched to the alcohol calories. This strategy is widely used to study end-organ injury, including chronic alcohol-induced skeletal muscle pathology (Levitt et al., 2020c; Crowel et al., 2016; Lang, 2018).

5) Oral gavage: Animals are orally administered alcohol via gavage. The oral gavage method is commonly used to study acute effects of a single dose of alcohol on muscle pathology or to administer ethanol at binge doses in combination with the Lieber-DeCarli diet; e.g. "binge-on-chronic" or "NIAAA model" (Samuelson et al., 2019; Bertola et al., 2013).

6) Systemic injection: This is generally accomplished using an intraperitoneal injection and is commonly used to assess acute effects of alcohol on skeletal muscle (Steiner and Lang, 2015; Steiner et al., 2016). 
7) Inhalation of alcohol vapor: Animals are placed in chambers and exposed to alcohol vapor for specific periods of time, often referred to as "intermittent ethanol exposure". This is a common method to make rodents dependent on alcohol (Gilpin et al., 2008; Mouton et al., 2016).

8) Genetic models: Mice or rats are bred to create lines of animals that are sensitive or insensitive to alcohol and rat lines have been generated to select for alcohol preference. For example, selectively-bred Sardinian alcohol-preferring (sP) and non-preferring (sNP) rats reliably exhibit strong alcohol preference and motivation (sP) or strong avoidance (sNP) of alcohol (Colombo et al., 2006). Other genetic models of alcoholrelated behaviors include inbred strains, recombinant inbred strains, and transgenic/ knock-out mice (Mayfield et al., 2016).

The metabolic rate of alcohol varies among species, and doses are optimized to generate blood and brain alcohol levels that produce pharmacological effects. For example, that rate of alcohol clearance is much greater in rats compared to humans and further varies among strains (Erickson, 1984; Holford, 1987). Because of the differences in rates of alcohol metabolism, while the method of alcohol administration may not exactly mimic human consumption, it serves to study the effects of alcohol on end organ injury.

Rodent models commonly used to study alcoholic myopathy are chronic LieberDeCarli diet feeding, oral gavage, or systemic injections. In addition, in vitro studies using established cell lines (e.g. C2C12 mousederived myoblasts or primary myoblasts) are used to mechanistically understand how alcohol affects myoblast proliferation, differentiation, metabolism, and function. Here, we will discuss key findings of alcohol-mediated effects on skeletal muscle reported from studies utilizing rodent models.

\section{Muscle stem cell regenerative capacity}

Rodent studies have demonstrated that chronic alcohol feeding increases skeletal muscle gene expression of TNFa and interleukin
(IL)-6, indicating chronic inflammation (Steiner and Lang, 2015a) . Our published work showed decreased differentiation potential of skeletal muscle stem cells isolated from chronic binge alcohol-administered macaques. This was associated with reduced expression of myogenic genes and impaired myotube formation, indicative of impaired muscle fiber formation (Simon et al., 2014; Simon et al., 2017). The marked dysregulation of myoblast myogenic and inflammatory gene expression and myotube formation with chronic alcohol administration reflects impaired muscle regenerative capacity and is likely to contribute to decreased muscle mass, especially in response to an injury or disuse atrophy. Alcohol and aging are risk factors for traumatic injury and subsequent immobilization-induced muscle atrophy (Lukaszyk et al., 2016). In fact, about $10-30 \%$ of hospitalized older patients have diagnosed AUD (O'Connell et al., 2003). Skeletal muscle recovery may be complicated by alcohol use and altered hormonal status among older individuals (Lukaszyk et al., 2016). To examine this question, we utilized LieberDeCarli feeding in rats for 10 weeks culminating in 1 week of unilateral hind limb immobilization followed by 3 or 14 days of remobilization (Levitt et al., 2020c). Our data indicated that alcohol dysregulates the expression of markers of muscle regeneration following unilateral hind limb immobilization. Although alcohol did not significantly exacerbate the immobilization-mediated decrease in muscle weight, it is possible that underlying differences in regeneration may have occurred Therefore, when immobilization is indicated, caution is warranted about alcohol use during the immobilization and post-immobilization recovery periods.

\section{Mitochondrial homeostasis and bioener- getics}

Alcohol impairs mitochondrial function in tissues including skeletal and cardiac muscle (Guo and Ren, 2010; Kumar et al., 2019; Duplanty et al., 2017; Duplanty et al., 2018), and mitochondrial homeostasis is critical in the maintenance of functional metabolic muscle mass (Romanello and Sandri, 2015). In chronic alcohol fed rats, decreased mitochondrial fusion and connectivity, calcium dysregulation, 
and impaired mitochondrial bioenergetics and excitation-contraction coupling have been reported (Eisner et al., 2014; Trounce et al., 1990). Studies also show that chronic alcohol decreases muscle cross sectional area with a decrease in total and free glutathione content, decreased glutathione reductase activity and decreased expression of oxidative stress genes (Otis and Guidot, 2010; Otis et al., 2007). Together, a number of studies have shown alcohol-mediated oxidative damage in muscle as evidenced by an increase in protein carbonyl, cholesterol hydroperoxide, and malondialdehyde content (Otis et al., 2007; Koo-Ng et al., 2000).

\section{Alcohol-mediated}

impaired bioenergetics are not limited to mitochondria. Chronic alcohol administration results in skeletal muscle glycolytic impairments in rodents. For example, activity of the glycolytic enzymes aldolase, pyruvate kinase, and lactate dehydrogenase were significantly decreased in vastus lateralis after chronic ethanol administration (Trounce et al., 1990), mirroring findings in humans (Trounce et al., 1987). In contrast, opposite effects have been observed in white gastrocnemius muscle in male rats, suggesting a potential fiber type specific effect of ethanol on glycolytic enzyme activities (Vila et al., 2001). Using transcriptomics, decreased expression of genes in the glycolytic pathway were also reported after $\mathrm{C} 2 \mathrm{C} 12$ myoblasts were treated with $100 \mathrm{mM}$ ethanol, a supraphysiological dose, for 6 or 24 hours (Kumar et al., 2019). Although these previous findings were at the transcriptomic or enzyme activity levels, we recently confirmed that 3 days of treatment with $50 \mathrm{mM}$ ethanol impairs glycolytic function in live myoblasts in vitro (Levitt et al., 2020).

\section{Skeletal muscle protein synthesis}

Clinical studies provide evidence that a major mechanism of alcohol-induced myopathy is altered balance of protein synthesis and breakdown (Steiner and Lang, 2015; Reilly et al., 2000; Steiner et al., 2015). To identify alcohol-induced changes in specific proteins in the protein synthesis and degradation pathway, liquid alcohol diets have been used.
The mammalian target of rapamycin (mTOR) pathway plays a central role in protein synthesis and is important for controlling skeletal muscle mass. mTOR activation activates two signaling pathways; S6 kinase 1 (S6K1) phosphorylation leading to activation of the ribosomal protein S6 and phosphorylation of the eukaryotic initiation factor 4E (elF4E)-binding protein (4EBP1) releasing its inhibition of the translation initiation factor elF-4E. Chronic alcohol consumption decreases phosphorylation of mTOR itself (Lang et al., 2003a), ribosomal protein S6 (rpS6), and phosphorylation of 4E-BP1 in skeletal muscle (Korzick et al., 2013). This is associated with a greater proportion of elF4E in the inactive elF4E-4EBP1 complex versus the active elF4E-elF4G and thus illustrates one possible mechanism by which alcohol decreases protein synthesis. Insulin and insulin-like growth factor (IGF)-1 are anabolic hormones that activate mTOR signaling. Acute ethanol markedly attenuated the insulin and IGF-1 mediated increases in S6K1 and rpS6 phosphorylation but did not simultaneously impair signaling through 4E-BP1 (Kumar et al., 2002). It should be noted that phosphorylation was measured at only one time point in that study, so it is possible that an acute effect was present at a different time point. However, ethanol attenuated the leucine-stimulated phosphorylation of 4E-BP1 and elF4G in addition to mTOR, S6K1, and rpS6 (Lang et al., 2003). In addition, rodent models show that alcohol significantly decreases IGF-1 levels in both plasma and muscle, and this decrease is correlated with decreased muscle protein synthesis (Lang et al., 1998). Overall, there is strong evidence that alcohol attenuates basal mTOR signaling after chronic administration, acutely attenuates mTOR signaling in response to anabolic stimulation, and may decrease systemic levels of the anabolic hormone, IGF-1.

\section{Skeletal muscle protein degradation}

Protein degradation in skeletal muscle is directed primarily by two pathways, the ubiquitin proteasome pathway (UPP) and the autophagic-lysosomal system (Steiner and Lang, 2015; Steiner et al., 2015; White et al., 2014). The two ubiquitin ligases or atrogenes, 
atrogin-1 and MuRF1 are specific to the muscle and are increased in several catabolic conditions. Both atrogin-1 and MURF1 are increased in acute and chronic alcohol administered rodents (Korzick et al., 2013; Vary et al., 2008; Vargas and Lang, 2008), possibly reflecting increased protein degradation activity. However, chronic alcohol did not further increase atrogin-1 and MURF1 expression or proteasomal activity in aged rats (Korzick et al., 2013). The autophagic-lysosomal system is activated due to cellular stress and mediates degradation of misfolded proteins. Whether alcohol-mediated muscle protein degradation is mediated by autophagy is still not clear. In alcohol-fed mice, there is increased expression of autophagy markers and in vitro treatment of C2C12 myotubes with $100 \mathrm{mM}$ alcohol (a supraphysiological level of alcohol) increased autophagic gene expression within $6 \mathrm{~h}$ (Thapaliya et al., 2014). However, others have not observed this change in chronic alcohol fed mice (Steiner and Lang, 2015). Similarly, our studies in primary myoblasts derived from chronic alcohol administered macaques do not show changes in autophagic markers compared to myoblasts derived from vehicle administered macaques (Simon et al., 2014). Thus, studies in rodent models suggest that alcohol-mediated decreased protein synthesis and increased protein degradation can potentially contribute to alcoholic myopathy.

\section{Skeletal muscle mass and morphology}

Decreased skeletal muscle mass is characteristic of chronic at-risk alcohol use, and structural abnormalities have been observed in muscle biopsy samples from such subjects (Fernandez-Sola et al., 2007). However, studying these phenomena in humans is complicated by differences in nutrition, current and past physical activity, hormonal status, etc. Therefore, rodent models have been employed to examine alcohol-mediated muscle wasting in controlled settings. For example, alcoholfed rats showed decreased lean body mass compared to pair-fed controls (Korzick et al., 2013). A further benefit of studying such effects in rodents is that many individual rodent muscles have a more homogenous muscle fiber type population (e.g. gastrocnemius is composed of $\sim 95 \%$ type II fibers) than in humans (e.g., gastrocnemius is composed of $\sim 50 \%$ type II fibers). The homogenous fiber type composition allows for easier assessment of the effects of alcohol on different fiber types. Chronic alcohol (15\% v/v in drinking water) decreased muscle fiber cross-sectional area across all fiber types in the plantaris, which is composed of $\sim 95 \%$ type II fibers in rats (Vila et al., 2001). In the same study, muscle fiber cross-sectional area was unaffected in the type I-dominant soleus and red gastrocnemius muscles. We observed decreased quadriceps mass (white and red portions together) in the non-immobilized hind limb of alcohol-fed rats compared to their pairfed counterparts (Levitt et al., 2020c). Without immobilization, type II-dominant plantaris mass and total protein were decreased in rats fed ethanol for 12 weeks (Clary et al., 2011). Ten weeks of alcohol feeding decreased total protein and RNA in type II-rich gastrocnemius and plantaris but not type I-dominant soleus, with concomitant decreases in expression of the structural proteins titin and nebulin in white gastrocnemius (Hunter et al., 2002), which could further contribute to alcoholic myopathy, particularly in type II muscle. Overall, results from rodent studies confirm that alcoholinduced muscle wasting more severely affects type II-dominant muscle and provide a viable model to elucidate underlying mechanisms.

\section{Skeletal muscle function}

Although skeletal muscle mass and function are generally related, a host of factors (bioenergetic, neurological etc.) confound this non-linear relationship. Even without sufficient time to decrease muscle mass, acute binge alcohol administration in humans after exerciseinduced muscle damage can exacerbate the exercise-induced decrease in force production in men (Barnes et al., 2010), although this effect does not appear to translate to women (Levitt et al., 2017), and is not apparent when participants are accustomed to the exercise (Levitt et al., $2020 b)$. For ethical purposes, the doses of alcohol used in human studies of acute binge drinking produce blood alcohol concentrations of only $\sim 0.08-0.12 \mathrm{~g} / \mathrm{dL}$. This may be why an additional stimulus (e.g. exercise-induced muscle damage) is needed to uncover effects. 
However, human consumption of ethanol can result in blood alcohol concentrations that far exceed those in these studies and may produce acute and cumulative effects on muscle function as muscle weakness is observed in approximately half of patients with AUD (Preedy et al., 2001). Rodent models have been used to examine acute and chronic effects of alcohol on muscle contractile function. In one such study, extensor digitorum longus (EDL) contractile properties post-fatigue were impaired after chronic ethanol feeding but not 2 hours after acute ethanol intoxication @ $3 \mathrm{~g} / \mathrm{kg}$ (Crowell et al., 2019). Previous work examining the acute effects of alcohol on muscle contractile function in rat EDL in vitro observed decreased twitch tension at higher ( 0.4 and $2.5 \mathrm{~g} \%$ ethanol) but not lower ( 0.1 and $0.2 \mathrm{~g} \%$ ethanol) ethanol doses (Taylor et al., 1992). These findings suggest dose dependent effects of alcohol on muscle function. Overall, more work is needed to elucidate effects of alcohol on skeletal muscle contractile function, and rodent models are well-suited for such studies.

\section{Conclusion}

Alcoholic myopathy is more prevalent than well-known organ injury like alcoholic hepatitis. Clinical studies in human subjects with AUD are challenging for many reasons including the presence of confounding lifestyle factors, comorbid conditions, and varying patterns and severity of alcohol consumption. Rodent models of alcohol administration have allowed for controlled studies to standardize administration across subjects and minimize confounding factors. The specific experimental question and study design parameters must be considered when selecting an alcohol administration protocol. The most used experimental paradigms to study alcohol-mediated muscle pathology are alcohol-containing liquid diets (chronic model) and intraperitoneal injections or oral gavage (acute models). Using these rodent models, many factors underlying alcoholic myopathy, including impaired regenerative capacity, altered anabolic/catabolic balance, impaired bioenergetic function, and skeletal muscle morphological and contractile changes have been described. Thus, mechanistic studies in rodent models have helped identify key alcoholmediated pathways that are dysregulated and, in the future, may provide therapeutic targets to ameliorate alcoholic myopathy.

\section{REFERENCES}

Barnes, M. J., Mündel, T. and Stannard, S. R. 2010. Post-exercise alcohol ingestion exacerbates eccentric-exercise induced losses in performance. Eur. J. Appl. Physiol. 108: 1009-1014.

Bertola, A., Mathews, S., Ki, S. H., Wang, H. and Gao, B. 2013. Mouse model of chronic and binge ethanol feeding (the NIAAA model). Nat. Protoc. 8: 627-637

Clary, C. R., Guidot, D. M., Bratina, M. A. and Otis, J. S. 2011. Chronic alcohol ingestion exacerbates skeletal muscle myopathy in HIV-1 transgenic rats. AIDS Res. Ther. 8: 30.

Colombo, G., Lobina, C., Carai, M. A. and Gessa, G. L. 2006. Phenotypic characterization of genetically selected Sardinian alcohol-preferring (sP) and -non-preferring (sNP) rats. Addict. Biol. 11: 324-338.

Crowell, K. T., Laufenberg, L. J. and Lang, C. H. 2019. Chronic alcohol consumption, but not acute intoxication, decreases in vitro skeletal muscle contractile function. Alcohol. Clin. Exp. Res. 43: 2090-2099.

Crowell, K. T., Steiner, J. L., Coleman, C. S. and Lang, C. H. 2016. Decreased wholebody fat mass produced by chronic alcohol consumption is associated with activation of S6K1-mediated protein synthesis and increased autophagy in epididymal white adipose tissue. Alcohol. Clin. Exp. Res. 40, 1832-1845.

Duplanty, A. A., Siggins, R. W., Allerton, T., Simon, L.and Molina, P.E.2018. Myoblast mitochondrial respiration is decreased in chronic binge alcohol administered simian immunodeficiency virus-infected antiretroviral-treated rhesus macaques. Physiol. Rep. 6: 13625. 
Duplanty, A. A., Simon, L. and Molina, P. E. 2017. Chronic binge alcohol-induced dysregulation of mitochondrial-related genes in skeletal muscle of simian immunodeficiency virus-infected rhesus macaques at end-stage disease. Alcohol Alcohol. 52: 298-304.

Eisner, V., Lenaers, G. and Hajnoczky, G. 2014. Mitochondrial fusion is frequent in skeletal muscle and supports excitationcontraction coupling. J. Cell Biol. 205: 179-195.

Erickson, C. K. 1984. Ethanol clearance in nine inbred rat strains. Alcohol Clin. Exp. Res. 8: 491-494.

Estruch, R., Nicolas, J. M., Villegas, E., Junque, A. and Urbano-Marquez, A. 1993. Relationship between ethanolrelated diseases and nutritional status in chronically alcoholic men. Alcohol and Alcoholism. 28: 543-550.

Fernandez-Sola, J., Preedy, V. R. , Lang, C. H., Gonzalez-Reimers, E., Arno, M., Lin, J. C., Wiseman, H., Zhou, S., Emery, P. W., Nakahara, T., Hashimoto, K., Hirano, M., Santolaria-Fernandez, F., GonzálezHernandez, T., Fatjo, F., Sacanella, E., Estruch, R., Nicolas, J. M. and UrbanoMarquez A. 2007. Molecular and cellular events in alcohol-induced muscle disease. Molecular and cellular events in alcohol-induced muscle disease. Alcohol. Clin. Exp. Res. 31: 1953-1962.

Gilpin, N. W., Richardson, H. N., Cole, M. and Koob, G. F. 2008. Vapor inhalation of alcohol in rats. Curr. Protoc. Neurosci. Chapter 9: Unit 9.29.

Grahame, N. J., Li, T. K. and Lumeng, L. 1999 > Selective breeding for high and low alcohol preference in mice. Behav. Genet. 29: 47-57.

Guo, R. and Ren, J. 2010. Alcohol dehydrogenase accentuates ethanolinduced myocardial dysfunction and mitochondrial damage in mice: role of mitochondrial death pathway. PLOS One. 5: e8757.
Jellinek, E. M. and McFarland, R. A. 1940. Analysis of psychological experiments on the effects of alcohol. Quarterly J. Studies on Alcohol. 1: 272-371.

Holford, N. H. 1987. Clinical pharmacokinetics of ethanol. Clin. Pharmacokinet. 13: 273-292.

Hunter, A. M., Gibson, A., Derman, W. E., Lambert, M., Dennis, S. C. and Noakes, T. D. 2002. The effect of selective beta1blockade on EMG signal characteristics during progressive endurance exercise. Eur. J. Appl. Physiol. 88: 275-281.

Koo-Ng, R., Falkous, G., Reilly, M., Peters, T. J., Mantle, D. and Preedy, V. R. 2000. Carbonyl levels in type I and II fiber-rich muscles and their response to chronic ethanol feeding in vivo and hydroxyl and superoxide radicals in vitro. Alcohol Clin. Exp. Res. 24: 1862-1868.

Korzick, D. H., Sharda, D. R., Pruznak, A. M. and Lang, C. H.2013. Aging accentuates alcohol-induced decrease in protein synthesis in gastrocnemius. Am. J. Physiol. Regul. Integr. Comp. Physiol. 304: R887-898.

Kumar, A., Davuluri, G., Welch, N., Kim, A., Gangadhariah, M., Allawy, A., Priyadarshini, A., McMullen, M. R., Sandlers, Y., Willard, B. and Hoppel, C. L. 2019. Oxidative stress mediates ethanolinduced skeletal muscle mitochondrial dysfunction and dysregulated protein synthesis and autophagy. Free Radic. Biol. Med. 145: 284-299.

Kumar, V., Frost, R. A. and Lang, C. H. 2002. Alcohol impairs insulin and IGF-I stimulation of S6K1 but not 4E-BP1 in skeletal muscle. Am. J. Physiol. Endocrinol. Metab. 283: E917-928

Lang, C. H. 2018. Lack of sexual dimorphism on the inhibitory effect of alcohol on muscle protein synthesis in rats under basal conditions and after anabolic stimulation. Physiol. Rep. 6: e13929. 
Lang, C. H., Fan, J., Lipton, B. P., Potter, B. J. and McDonough, K.H. 1998. Modulation of the insulin-like growth factor system by chronic alcohol feeding. Alcohol Clin. Exp. Res. 22: 823-829.

Lang, C. H., Frost, R. A, Sumner, A. D and Vary, T. C. 2005. Molecular mechanisms responsible for alcohol-induced myopathy in skeletal muscle and heart. Int. J. Biochem. Cell Biol. 37: 21802195.

Lang, C. H., Kumar, V., Liu, X., Frost, R. A. and Vary, T. C. 2003. IGF-I induced phosphorylation of S6K1 and 4E-BP1 in heart is impaired by acute alcohol intoxication. Alcohol Clin. Exp. Res. 27: 485-494.

Lang, C. H., Vary, T. C. and Frost, R. A. 2003a. Acute in vivo elevation of insulin-like growth factor (IGF) binding protein-1 decreases plasma free IGF-I and muscle protein synthesis. Endocrinol. 144: 3922-3933.

Levitt, D. E., Chalapati, N., Prendergast, M. J., Simon, L. and Molina, P. E. 2020a. Ethanol-impaired myogenic differentiation is associated with decreased myoblast glycolytic function. Alcohol Clin. Exp. Res. 44: 2166-2176.

Levitt, D. E., Idemudia, N. O., Cregar, C. M., Duplanty, A. A., Hill, D. W. and Vingren, J. L., 2020b. Alcohol after resistance exercise does not affect muscle power recovery. J. Strength Cond. Res. 34: 1938-1944.

Levitt, D. E., Luk, H.Y., Duplanty, A. A., McFarlin, B. K., Hill, D. W. and Vingren, J. L. 2017. Effect of alcohol after muscle-damaging resistance exercise on muscular performance recovery and inflammatory capacity in women. Eur. J. Appl. Physiol. 117: 1195-1206.

Levitt, D.E., Yeh, A.Y., Prendergast, M.J., Budnar Jr, R.G., Adler, K.A., Cook, G., Molina, P.E. and Simon, L., 2020c. Chronic alcohol dysregulates skeletal muscle myogenic gene expression after hind limb immobilization in female rats. Biomolecules. 10: 441

Lieber, C. S. and DeCarli, L. M. Liquid diet technique of ethanol administration: 1989 update. 1989. Alcohol Alcohol. 24: 197-211.

Lukaszyk, C., Harvey, L., Sherrington, C., Keay, L., Tiedemann, A., Coombes, J., Clemson, L. and Ivers, R., 2016. Risk factors, incidence, consequences and prevention strategies for falls and fall-injury within older indigenous populations: a systematic review. Aust. NZJ Public Health. 40: 564-568.

Martin, F., Ward, K., Slavin, G., Levi, J. and Peters, T. J. 1985. Alcoholic skeletal myopathy, a clinical and pathological study. QJM, An International Journal of Medicine. 55: 233-251.

Mayfield, J., Arends, M. A., Harris, R. A. and Blednov, Y. A. 2016. Genes and alcohol consumption: studies with mutant mice. Int. Rev. Neurobiol. 126: 293-355.

Mouton, A.J., Maxi, J.K., Souza-Smith, F., Bagby, G.J., Gilpin, N.W., Molina, P.E. and Gardner, J.D., 2016. Alcohol vapor inhalation as a model of alcohol-induced organ disease. Alcohol, Clin. Exp. Res. 40: 1671-1678.

Nentwig, T. B., Starr, E. M., Chandler, L. J. and Glover, E. J. 2019. Absence of compulsive drinking phenotype in adult male rats exposed to ethanol in a bingelike pattern during adolescence. Alcohol. 79: 93-103.

O'Connell, H., Chin, A. V., Cunningham, C. and Lawlor, B. 2003. Alcohol use disorders in elderly people--redefining an age old problem in old age. BMJ. 327: 664-667.

Otis, J. S., Brown, L. A. and Guidot, D. M. 2007. Oxidant-induced atrogin-1 and transforming growth factor-beta1 precede alcohol-related myopathy in rats. Muscle Nerve 36, 842-848. 
Otis, J. S. and Guidot, D. M. 2010. Procysteine increases alcohol-depleted glutathione stores in rat plantaris following a period of abstinence. Alcohol Alcohol. 45: 495500.

Pahng, A.R., Paulsen, R.I., McGinn, M.A., Farooq, M.A., Edwards, K.N. and Edwards, S. 2019. Alcohol. 75: 11-18.

Preedy, V. R., Adachi, J., Ueno, Y., Ahmed, S., Mantle, D., Mullatti, N., Rajendram, R. and Peters, T. J., 2001. Alcoholic skeletal muscle myopathy: definitions, features, contribution of neuropathy, impact and diagnosis. Eur. J. Neurol. 8: 677-687.

Preedy, V.R., Ohlendieck, K., Adachi, J., Koll, M., Sneddon, A., Hunter, R., Rajendram, R., Mantle, D. and Peters, T.J., 2003. The importance of alcohol-induced muscle disease. J. Muscle Res.Cell Motility. 24: pp.55-63.

Reilly, M. E., Patel, V. B., Peters, T. J. and Preedy, V. R. 2000. In vivo rates of skeletal muscle protein synthesis in rats are decreased by acute ethanol treatment but are not ameliorated by supplemental alpha-tocopherol. The J. Nutr. 130 : 3045-3049.

Romanello, V. and Sandri, M. 2015. Mitochondrial quality control and muscle mass maintenance. Front. Physiol. 6: 422.

Samuelson, D. R., Siggins, R. W., Ruan, S., Amedee, A. M., Sun, J., Zhu, Q. K., Marasco, W. A., Taylor, C. M., Luo, M., Welsh, D. A. and Shellito, J. E., 2019. Alcohol consumption increases susceptibility to pneumococcal pneumonia in a humanized murine HIV model mediated by intestinal dysbiosis. Alcohol. 80:33-43.

Simon, L., Ford Jr, S. M., Song, K., Berner, P., Vande Stouwe, C., Nelson, S., Bagby, G. J. and Molina, P. E., 2017. Decreased myoblast differentiation in chronic binge alcohol-administered simian immunodeficiency virus-infected male macaques: role of decreased miR206. Am. J. Physiol. Reg. Integr. Comp. Physiol. 313: R240-R250.

Simon, L., LeCapitaine, N., Berner, P., Vande Stouwe, C., Mussell, J.C., Allerton, T., Primeaux, S. D., Dufour, J., Nelson, S., Bagby, G. J. and Cefalu, W., 2014. Chronic binge alcohol consumption alters myogenic gene expression and reduces in vitro myogenic differentiation potential of myoblasts from rhesus macaques. Am. J. Physiol. Reg. Integr. Comp. Physiol. 306: R837-844.

Steiner, J. L., Gordon, B. S. and Lang, C.H. 2015. Moderate alcohol consumption does not impair overload-induced muscle hypertrophy and protein synthesis. Physiol. Rep. 3: 12333.

Steiner, J. L., Kimball, S. R. and Lang, C. H 2016. Acute alcohol-induced decrease in muscle protein synthesis in female mice is REDD-1 and mTOR-independent. Alcohol Alcohol. 51: 242-250.

Steiner, J. L. and Lang, C. H. 2015. Alcohol intoxication following muscle contraction in mice decreases muscle protein synthesis but not mTOR signal transduction. Alcohol. Clin. Exp. Res. 39: 1-10.

Steiner, J. L. and Lang, C. H. 2015a Dysregulation of skeletal muscle protein metabolism by alcohol. Am. J. Physiol. Endocrinol. Metab. 308: E699-712.

Stielper, Z.F., Fucich, E.A., Middleton, J.W., Hillard, C.J., Edwards, S., Molina, P.E. and Gilpin, N.W. 2021. Traumatic Brain Injury and Alcohol Drinking Alter Basolateral Amygdala Endocannabinoids in Female Rats. J. Neurotrauma, 38: 422-434.

Taylor, R. G., Abresch, R. T., Lieberman, J. S., Sharman, R. B., Entrikin, R. K. and Fowler Jr, W. M., 1992. Fast and slow skeletal muscles: effect of ethanol on contractility of muscles from mice. Arch. Phys. Med. Rehabil. 73: 355-358. 
Thapaliya, S., Runkana, A., McMullen, M. R., Nagy, L. E., McDonald, C., Prasad, S. V. N. and Dasarathy, S., 2014. Alcoholinduced autophagy contributes to loss in skeletal muscle mass. Autophagy. 10: 677-690.

Trounce, I., Byrne, E. and Dennett, X. 1990. Biochemical and morphological studies of skeletal muscle in experimental chronic alcoholic myopathy. Acta Neurol. Scand. 82: 386-391.

Trounce, I., Byrne, E., Dennett, X., Santamaria, J., Doery, J. and Peppard, R. 1987. Chronic alcoholic proximal wasting: physiological, morphological and biochemical studies in skeletal muscle. Aust. NZJ Med. 17: 413-419.

Tsukamoto, H., Gaal, K. and French, S. W. Insights into the pathogenesis of alcoholic liver necrosis and fibrosis: status report. 1990. Hepatology. 12: 599-608.

Vargas, R. and Lang, C. H. 2008. Alcohol accelerates loss of muscle and impairs recovery of muscle mass resulting from disuse atrophy. Alcohol. Clin. Exp. Res. 32: 128-137.
Vary, T. C., Frost, R. A. and Lang, C. H. 2008. Acute alcohol intoxication increases atrogin-1 and MuRF1 mRNA without increasing proteolysis in skeletal muscle. Am. J. Physiol. Reg. Integr. Comp. Physiol. 294: R1777-1789.

Vila, L., Ferrando, A., Voces, J., de Oliveira, C.C., Prieto, J.G. and Alvarez, A.I. 2001. Effect of chronic ethanol ingestion and exercise training on skeletal muscle in rat. Drug Alcohol Depend. 64: 27-33.

White, P.J., St-Pierre, P., Charbonneau, A., Mitchell, P.L., St-Amand, E., Marcotte, B. and Marette, A. 2014. Protectin DX alleviates insulin resistance by activating a myokine-liver glucoregulatory axis. Nat. Med. 20: 664-669.

Younis, R. M., Wolstenholme, J. T., Bagdas, D., Bettinger, J. C., Miles, M. F. and Damaj, M. I. 2019. Adolescent but not adult ethanol binge drinking modulates ethanol behavioral effects in mice later in life. Pharmacol. Biochem. Behav. 184: p172740. 